\title{
Parallel Methods and Higher Dimensional NLS Equations
}

\author{
M. S. Ismail ${ }^{1}$ and T. R. Taha ${ }^{2}$ \\ ${ }^{1}$ Department of Mathematics, College of Science, King Abdulaziz University, P.O. Box 80203, Jeddah, Saudi Arabia \\ ${ }^{2}$ Department of Computer Science, University of Georgia, Athens, GA 30602-7404, USA
}

Correspondence should be addressed to M. S. Ismail; msismail@kau.edu.sa

Received 3 June 2013; Accepted 28 July 2013

Academic Editor: Juan Carlos Cortés López

Copyright (C) 2013 M. S. Ismail and T. R. Taha. This is an open access article distributed under the Creative Commons Attribution License, which permits unrestricted use, distribution, and reproduction in any medium, provided the original work is properly cited.

\begin{abstract}
Alternating direction implicit (ADI) schemes are proposed for the solution of the two-dimensional coupled nonlinear Schrödinger equation. These schemes are of second- and fourth-order accuracy in space and second order in time. The resulting schemes in each ADI computation step correspond to a block tridiagonal system which can be solved by using one-dimensional block tridiagonal algorithm with a considerable saving in computational time. These schemes are very well suited for parallel implementation on a high performance system with many processors due to the nature of the computation that involves solving the same block tridiagonal systems with many right hand sides. Numerical experiments on one processor system are conducted to demonstrate the efficiency and accuracy of these schemes by comparing them with the analytic solutions. The results show that the proposed schemes give highly accurate results.
\end{abstract}

\section{Introduction}

In this paper, we consider the coupled nonlinear Schrödinger equation

$$
\begin{array}{r}
i \psi_{t}+\delta\left(\psi_{x x}+\psi_{y y}\right)+\left(|\psi|^{2}+\alpha|\phi|^{2}\right) \psi=0, \\
(x, y, t) \in \Omega \times(0, T], \\
i \phi_{t}+\delta\left(\phi_{x x}+\phi_{y y}\right)+\left(\alpha|\psi|^{2}+|\phi|^{2}\right) \phi=0
\end{array}
$$

with initial conditions

$$
\begin{array}{r}
\psi(x, y, 0)=f_{1}(x, y), \quad \phi(x, y, 0)= \\
f_{2}(x, y), \\
(x, y) \in \Omega
\end{array}
$$

and Dirichlet boundary conditions

$$
\begin{array}{r}
\psi(x, y, t)=g_{1}(x, y, t), \quad \phi(x, y, t)=g_{2}(x, y, t), \\
(x, y, t) \in \partial \Omega \times(0, T],
\end{array}
$$

where $\Omega$ is a rectangular domain in $\mathbb{R}^{2}$. We assume that $\Omega=[a, b] \times[c, d], \partial \Omega$ is the boundary of $\Omega,(0, T]$ is the time interval, and $f_{1}(x, y), f_{2}(x, y), g_{1}(x, y, t)$, and $g_{2}(x, y, t)$ are given sufficiently smooth functions. The two functions $\psi(x, y, t), \phi(x, y, t)$ are representing the amplitudes of the two circularly polarized waves. The values of $\alpha$ vary over a wide range; that is, $\alpha \geq 2 / 3$ and $\alpha \leq 7$ correspond to kerr type electronic nonlinearity [1]. The physical significance of system (1) can be seen in the transverse effects in nonlinear optics. Since solitons interact like particles, the studies for the interactions of solitons have been of both experimental and theoretical interest. Many numerical methods have been developed for solving the coupled nonlinear Schrödinger [26]. Many published works for solving the two-dimensional nonlinear Schrödinger equation are given in [7-11]. In this work, we are going to derive an ADI method for solving the two-dimensional coupled nonlinear Schrödinger equation.

In this paper, we derive two ADI schemes for solving the two-dimensional coupled nonlinear Schrödinger system, one is of second order in space and time directions, and the other one is of fourth order in space and second order in time. Both methods are producing a block nonlinear tridiagonal system; a fixed point method has been developed to solve this system. The proposed schemes are unconditionally stable using Fourier stability analysis.

The ADI method [8-15], which replaces the solution of multidimensional problems by sequences of one-dimen- 
sional cases, only needs to solve tridiagonal linear system or block tridiagonal systems, and the resulting schemes are unconditionally stable and received much attention in recent years.

Following Biswas [16], we derive the soliton solution of the system (1) which can be written as

$$
\begin{aligned}
& \psi(x, y, t) \\
& \quad=A \operatorname{sech}\left(\beta_{1} x+\beta_{2} y-v t\right) \exp \left[i\left(-k_{1} x-k_{2} y+\omega t+\theta\right)\right],
\end{aligned}
$$

$$
\begin{aligned}
& \phi(x, y, t) \\
& \quad=B \operatorname{sech}\left(\beta_{1} x+\beta_{2} y-v t\right) \exp \left[i\left(-k_{1} x-k_{2} y+\omega t+\theta\right)\right],
\end{aligned}
$$

where

$$
\begin{gathered}
\omega=\left[\delta\left(\beta_{1}^{2}+\beta_{2}^{2}\right)-\left(k_{1}^{2}+k_{2}^{2}\right)\right], \quad v=-2 \delta\left(k_{1} \beta_{1}+k_{2} \beta_{2}\right) \\
A=B=\left[\frac{2 \delta\left(\beta_{1}^{2}+\beta_{2}^{2}\right)}{(1+\alpha)}\right]^{1 / 2},
\end{gathered}
$$

and $\alpha, \beta_{1}, \beta_{2}, k_{1}$, and $k_{2}$ are arbitrary constants (see the appendix). The system has the conserved quantities [15]

$$
\begin{aligned}
& I_{1}=\iint_{-\infty}^{\infty}|\psi|^{2} d x d y=\text { constant } \\
& I_{2}=\iint_{-\infty}^{\infty}|\phi|^{2} d x d y=\text { constant. }
\end{aligned}
$$

To avoid the complex computation, we assume

$$
\begin{aligned}
& \psi=u_{1}+i u_{2}, \\
& \phi=u_{3}+i u_{4} .
\end{aligned}
$$

The system (1) can be written as

$$
\begin{aligned}
& \frac{\partial u_{1}}{\partial t}+\delta\left(\frac{\partial^{2} u_{2}}{\partial x^{2}}+\frac{\partial^{2} u_{2}}{\partial y^{2}}\right)+F(\mathbf{u}) u_{2}=0, \\
& \frac{\partial u_{2}}{\partial t}-\delta\left(\frac{\partial^{2} u_{1}}{\partial x^{2}}+\frac{\partial^{2} u_{1}}{\partial y^{2}}\right)-F(\mathbf{u}) u_{1}=0, \\
& \frac{\partial u_{3}}{\partial t}+\delta\left(\frac{\partial^{2} u_{4}}{\partial x^{2}}+\frac{\partial^{2} u_{4}}{\partial y^{2}}\right)+G(\mathbf{u}) u_{4}=0, \\
& \frac{\partial u_{4}}{\partial t}-\delta\left(\frac{\partial^{2} u_{3}}{\partial x^{2}}+\frac{\partial^{2} u_{3}}{\partial y^{2}}\right)-G(\mathbf{u}) u_{3}=0,
\end{aligned}
$$

where

$$
\begin{aligned}
& F(\mathbf{u})=u_{1}^{2}+u_{2}^{2}+\alpha\left(u_{3}^{2}+u_{4}^{2}\right), \\
& G(\mathbf{u})=\alpha\left(u_{1}^{2}+u_{2}^{2}\right)+u_{3}^{2}+u_{4}^{2} .
\end{aligned}
$$

System (10) can be written in matrix vector form as

$$
\frac{\partial \mathbf{u}}{\partial t}+\delta A\left(\frac{\partial^{2} \mathbf{u}}{\partial x^{2}}+\frac{\partial^{2} \mathbf{u}}{\partial y^{2}}\right)+B(\mathbf{u}) \mathbf{u}=\mathbf{0}
$$

where

$$
\begin{gathered}
A=\left[\begin{array}{cccc}
0 & 1 & 0 & 0 \\
-1 & 0 & 0 & 0 \\
0 & 0 & 0 & 1 \\
0 & 0 & -1 & 0
\end{array}\right], \quad B=\left[\begin{array}{cccc}
0 & f_{1} & 0 & 0 \\
-f_{1} & 0 & 0 & 0 \\
0 & 0 & 0 & f_{2} \\
0 & 0 & -f_{2} & 0
\end{array}\right], \\
f_{1}=\left(u_{1}^{2}+u_{2}^{2}\right)+\alpha\left(u_{3}^{2}+u_{4}^{2}\right), \\
f_{2}=\alpha\left(u_{1}^{2}+u_{2}^{2}\right)+\left(u_{3}^{2}+u_{4}^{2}\right) .
\end{gathered}
$$

The paper is organized as follows. In Section 2 we give two ADI schemes for solving the two-dimensional coupled nonlinear Schrödinger equation, and in Section 3, we present the von Neumann stability analysis for the proposed schemes. Numerical experiments for several problems are presented in Section 4. A parallel algorithm for the proposed ADI schemes is given in Section 5. Conclusions are given in Section 6.

\section{Numerical Method}

To derive the numerical schemes for solving system (1), we consider the domain of interest $\Omega=[a, b] \times[a, b]$, such that $(x, y) \in \Omega$. The domain is divided by a uniform mesh in each direction such that

$$
\begin{gathered}
x_{l}=a+l h, \quad l=0,1,2, \ldots, L, \\
y_{m}=a+m h, \quad m=0,1,2, \ldots, M, \\
t_{n}=n k, \quad n=0,1,2, \ldots, N,
\end{gathered}
$$

where $h$ and $k$ are the space and time step sizes, respectively. We denote $u_{l, m}^{n}$ and $U_{l, m}^{n}$ to be the exact and the numerical solutions at the point $\left(x_{l}, y_{m}, t_{n}\right)$, respectively.

2.1. Second-Order ADI Method. To derive the first scheme, we approximate the space derivative using the central difference formulae

$$
\begin{aligned}
\frac{\partial^{2} u}{\partial x^{2}} & =\frac{1}{h^{2}} \delta_{x}^{2} u_{l, m}+O\left(h^{2}\right) \\
& =\frac{1}{h^{2}}\left(u_{l+1, m}-2 u_{l, m}+u_{l-1, m}\right)+O\left(h^{2}\right) \\
\frac{\partial^{2} u}{\partial y^{2}} & =\frac{1}{h^{2}} \delta_{y}^{2} u_{l, m}+O\left(h^{2}\right) \\
& =\frac{1}{h^{2}}\left(u_{l, m+1}-2 u_{l, m}+u_{l, m-1}\right)+O\left(h^{2}\right) .
\end{aligned}
$$

We apply (15) to system (12); this will lead us to the following first order differential system in time

$$
\mathbf{u}_{t}+\frac{1}{h^{2}} A\left[\delta_{x}^{2}+\delta_{y}^{2}\right] \mathbf{u}_{l, m}+B\left(\mathbf{u}_{l, m}\right) \mathbf{u}_{l, m}=\mathbf{0} .
$$


Equation (16) is of second order in space.

By applying the Crank-Nicolson method for the temporal discretization, we get the following difference scheme with accuracy $O\left(k^{2}+h^{2}\right)$ :

$$
\begin{aligned}
\frac{1}{k}\left[\mathbf{U}_{l, m}^{n+1}\right. & \left.-\mathbf{U}_{l, m}^{n}\right] \\
& +\frac{1}{2 h^{2}} A\left[\delta_{x}^{2}+\delta_{y}^{2}\right]\left[\mathbf{U}_{l, m}^{n+1}+\mathbf{U}_{l, m}^{n}\right]+B(\overline{\mathbf{U}}) \overline{\mathbf{U}}=\mathbf{0},
\end{aligned}
$$

where

$$
\overline{\mathbf{U}}=\frac{1}{2}\left[\mathbf{U}_{l, m}^{n+1}+\mathbf{U}_{l, m}^{n}\right] .
$$

This scheme can be written as

$$
\begin{aligned}
& {\left[I+\frac{1}{2} r A \delta_{x}^{2}+\frac{1}{2} r A \delta_{y}^{2}\right] \mathbf{U}_{l, m}^{n+1}} \\
& \quad=\left[I-\frac{1}{2} r A \delta_{x}^{2}-\frac{1}{2} r A \delta_{y}^{2}\right] \mathbf{U}_{l, m}^{n}-B(\overline{\mathbf{U}}) \overline{\mathbf{U}}
\end{aligned}
$$

which can be approximated by using the factored form

$$
\begin{aligned}
{\left[I+\frac{1}{2} r A \delta_{x}^{2}\right]\left[I+\frac{1}{2} r A \delta_{y}^{2}\right] \mathbf{U}_{l, m}^{n+1} } \\
=\left[I-\frac{1}{2} r A \delta_{x}^{2}\right]\left[I-\frac{1}{2} r A \delta_{y}^{2}\right] \mathbf{U}_{l, m}^{n} \\
\quad-k B(\overline{\mathbf{U}}) \overline{\mathbf{U}}+\frac{r^{2}}{4}\left\{A \delta_{x}^{2}\right\}\left\{A \delta_{y}^{2}\right\}\left(\mathbf{U}_{l, m}^{n+1}-\mathbf{U}_{l, m}^{n}\right) .
\end{aligned}
$$
as

By Taylor's expansion, the last term in (20) can be written

$$
\begin{aligned}
\frac{r^{2}}{4}\left\{A \delta_{x}^{2}\right\} & \left\{A \delta_{y}^{2}\right\}\left(\mathbf{U}_{l, m}^{n+1}-\mathbf{U}_{l, m}^{n}\right) \\
= & -\frac{k^{2}}{4} \frac{\delta_{x}^{2} \delta_{x}^{2}}{h^{4}}\left(k\left(\frac{\partial u}{\partial t}\right)+O\left(k^{3}\right)\right) \\
= & -\frac{k^{3}}{4}\left[\frac{\partial^{5} u}{\partial^{2} x \partial^{2} y \partial t}\right]+O\left(k^{3} h^{2}\right)
\end{aligned}
$$

which is of the same order of accuracy of the truncation error, and we ignore it to obtain

$$
\begin{aligned}
{[I+} & \left.\frac{1}{2} r A \delta_{x}^{2}\right]\left[I+\frac{1}{2} r A \delta_{y}^{2}\right] \mathbf{U}_{l, m}^{n+1} \\
& =\left[I-\frac{1}{2} r A \delta_{x}^{2}\right]\left[I-\frac{1}{2} r A \delta_{y}^{2}\right] \mathbf{U}_{l, m}^{n}-k B(\overline{\mathbf{U}}) \overline{\mathbf{U}} .
\end{aligned}
$$

By introducing a new intermediate vector $\mathbf{U}_{l, m}^{*}$, we propose a D'Yakonov [12, 17] ADI-like scheme for the coupled system

$$
\begin{gathered}
{\left[I+\frac{1}{2} r A \delta_{x}^{2}\right] \mathbf{U}_{l, m}^{*}} \\
=\left[I-\frac{1}{2} r A \delta_{x}^{2}\right]\left[I-\frac{1}{2} r A \delta_{y}^{2}\right] \mathbf{U}_{l, m}^{n}-k B(\overline{\mathbf{U}}) \overline{\mathbf{U}} \\
{\left[I+\frac{1}{2} r A \delta_{y}^{2}\right] \mathbf{U}_{l, m}^{n+1}=\mathbf{U}_{l, m}^{*}}
\end{gathered}
$$

which is a nonlinear scheme. An iterative algorithm of fixed point nature can be used to solve the system of the nonlinear equations (23)-(24). The fixed point that we propose can be given by

$$
\begin{gathered}
{\left[I+\frac{1}{2} r A \delta_{x}^{2}\right] \mathbf{U}_{l, m}^{*}=\left[I-\frac{1}{2} r A \delta_{x}^{2}\right]\left[I-\frac{1}{2} r A \delta_{y}^{2}\right] \mathbf{U}_{l, m}^{n}} \\
-k B\left(\frac{\mathbf{U}_{l, m}^{n+1, s}+\mathbf{U}_{l, m}^{n}}{2}\right) \\
\times \frac{\left[\mathbf{U}_{l, m}^{n+1, s}+\mathbf{U}_{l, m}^{n}\right]}{2}, \\
{\left[I+\frac{1}{2} r A \delta_{y}^{2}\right] \mathbf{U}_{l, m}^{n+1, s+1}=\mathbf{U}_{l, m}^{*},}
\end{gathered}
$$

where the superscript $s$ denotes the $s$ th iterate for solving the nonlinear system of equations for each time step. The block tridiagonal matrix equations of (25) can be solved by Crout's method. The initial iterate $\mathbf{U}_{l, m}^{n+1,0}$ is chosen as

$$
\mathbf{U}_{l, m}^{n+1,0}=\mathbf{U}_{l, m}^{n} .
$$

The iteration continues until the condition

$$
\left\|\mathbf{U}_{l, m}^{n, s+1}-\mathbf{U}_{l, m}^{n, s}\right\|_{\infty} \leq 10^{-6}
$$

is satisfied.

The boundary value of the intermediate variable $\dot{\mathbf{U}}_{l, m}$ can be extracted from (24) and is given by the following formulas:

$$
\begin{gathered}
\mathbf{U}_{0, m}^{*}=\left[I+\frac{r}{2} A \delta_{y}^{2}\right] \mathbf{U}_{0, m}^{n+1}, \\
\mathbf{U}_{L+1, m}^{*}=\left[I+\frac{r}{2} A \delta_{y}^{2}\right] \mathbf{U}_{L, m}^{n+1} .
\end{gathered}
$$

2.2. Fourth-Order ADI Method. Now, we want to derive a highly accurate fourth order ADI scheme; to do this, we approximate the space derivatives by the following formulas

$$
\begin{aligned}
& \frac{\partial^{2} u}{\partial x^{2}}=\frac{1}{h^{2}} \frac{\delta_{x}^{2}}{1+(1 / 12) \delta_{x}^{2}} \mathbf{u}_{l, m}+O\left(h^{4}\right), \\
& \frac{\partial^{2} u}{\partial y^{2}}=\frac{1}{h^{2}} \frac{\delta_{y}^{2}}{1+(1 / 12) \delta_{y}^{2}} \mathbf{u}_{l, m}+O\left(h^{4}\right) .
\end{aligned}
$$

By using these approximations together with CrankNicolson for the time direction, we get the numerical scheme

$$
\begin{gathered}
\frac{1}{k}\left[\mathbf{U}_{l, m}^{n+1}-\mathbf{U}_{l, m}^{n}\right]+\frac{1}{2 h^{2}} A\left[\frac{\delta_{x}^{2}}{1+(1 / 12) \delta_{x}^{2}}+\frac{\delta_{y}^{2}}{1+(1 / 12) \delta_{y}^{2}}\right] \\
\times\left[\mathbf{U}_{l, m}^{n+1}+\mathbf{U}_{l, m}^{n}\right]+B(\overline{\mathbf{U}}) \overline{\mathbf{U}}=\mathbf{0}
\end{gathered}
$$


which can be written as

$$
\begin{aligned}
{[I+} & \left.\frac{r}{2} A\left(\frac{\delta_{x}^{2}}{1+(1 / 12) \delta_{x}^{2}}+\frac{\delta_{y}^{2}}{1+(1 / 12) \delta_{y}^{2}}\right)\right] \mathbf{U}_{l, m}^{n+1} \\
= & {\left[I-\frac{r}{2} A\left(\frac{\delta_{x}^{2}}{1+(1 / 12) \delta_{x}^{2}}+\frac{\delta_{y}^{2}}{1+(1 / 12) \delta_{y}^{2}}\right)\right] \mathbf{U}_{l, m}^{n} } \\
& -k B(\overline{\mathbf{U}}) \overline{\mathbf{U}},
\end{aligned}
$$

and this can be written in the factored form as

$$
\begin{aligned}
{\left[I+\frac{r}{2}\right.} & \left.A\left(\frac{\delta_{x}^{2}}{1+(1 / 12) \delta_{x}^{2}}\right)\right] \\
& \times\left[I+\frac{r}{2} A\left(\frac{\delta_{y}^{2}}{1+(1 / 12) \delta_{y}^{2}}\right)\right] \mathbf{U}_{l, m}^{n+1} \\
= & {\left[I-\frac{r}{2} A\left(\frac{\delta_{x}^{2}}{1+(1 / 12) \delta_{x}^{2}}\right)\right] } \\
& \times\left[I-\frac{r}{2} A\left(\frac{\delta_{y}^{2}}{1+(1 / 12) \delta_{y}^{2}}\right)\right] \mathbf{U}_{l, m}^{n}-k B(\overline{\mathbf{U}}) \overline{\mathbf{U}}
\end{aligned}
$$

The difference between (31) and (32) is

$$
\begin{aligned}
-\frac{r^{2}}{4} & {\left[\frac{\delta_{x}^{2}}{1+(1 / 12) \delta_{x}^{2}}\right]\left[\frac{\delta_{y}^{2}}{1+(1 / 12) \delta_{y}^{2}}\right]\left(\mathbf{U}_{l, m}^{n+1}-\mathbf{U}_{l, m}^{n}\right) } \\
& =-\frac{k^{2}}{4 h^{4}}\left[\frac{\delta_{x}^{2}}{1+(1 / 12) \delta_{x}^{2}}\right]\left[\frac{\delta_{y}^{2}}{1+(1 / 12) \delta_{y}^{2}}\right] \\
& \times\left(k\left(\frac{\partial u}{\partial t}\right)+O\left(k^{3}\right)\right) \\
& =-\frac{k^{3}}{4}\left[\frac{\partial^{5} u}{\partial^{2} x \partial^{2} y \partial t}\right]+O\left(k^{3} h^{4}\right)
\end{aligned}
$$

which is of the same order of accuracy of the truncation error, we ignore it, and then we operate on both sides of (32) by $\left(1+(1 / 12) \delta_{x}^{2}\right)\left(1+(1 / 12) \delta_{y}^{2}\right)$

$$
\begin{aligned}
{[(1+} & \left.\left.\frac{1}{12} \delta_{x}^{2}\right) I+\frac{r}{2} A \delta_{x}^{2}\right]\left[\left(1+\frac{1}{12} \delta_{y}^{2}\right) I+\frac{r}{2} A \delta_{y}^{2}\right] \mathbf{U}_{l, m}^{n+1} \\
= & {\left[\left(1+\frac{1}{12} \delta_{x}^{2}\right) I-\frac{r}{2} A \delta_{x}^{2}\right] } \\
& \times\left[\left(1+\frac{1}{12} \delta_{y}^{2}\right) I-\frac{r}{2} A \delta_{y}^{2}\right] \mathbf{U}_{l, m}^{n} \\
& -k\left(1+\frac{1}{12} \delta_{x}^{2}\right)\left(1+\frac{1}{12} \delta_{y}^{2}\right) B(\overline{\mathbf{U}}) \overline{\mathbf{U}}
\end{aligned}
$$

The fourth-order D'Yakonov ADI-like scheme in this case can be displayed as

$$
\begin{aligned}
& {\left[\left(1+\frac{1}{12} \delta_{x}^{2}\right) I+\frac{r}{2} A \delta_{x}^{2}\right] \dot{\mathbf{U}}} \\
& =\left[\left(1+\frac{1}{12} \delta_{x}^{2}\right) I-\frac{r}{2} A \delta_{x}^{2}\right]\left[\left(1+\frac{1}{12} \delta_{y}^{2}\right) I-\frac{r}{2} A \delta_{y}^{2}\right] \mathbf{U}_{l, m}^{n} \\
& \quad-k\left(1+\frac{1}{12} \delta_{x}^{2}\right)\left(1+\frac{1}{12} \delta_{y}^{2}\right) B(\overline{\mathbf{U}}) \overline{\mathbf{U}} \\
& \quad\left[\left(1+\frac{1}{12} \delta_{y}^{2}\right) I+\frac{r}{2} A \delta_{y}^{2}\right] \mathbf{U}_{l, m}^{n+1}=\dot{\mathbf{U}}
\end{aligned}
$$

Now the systems in (35) and (36) are nonlinear. By the similar approach used in the previous scheme, we can derive a fixed point iterative formulas.

The boundary value of the intermediate variable $\dot{\mathbf{U}}_{l, m}$ in (35) can be given by the following formulas:

$$
\begin{gathered}
\dot{\mathbf{U}}_{0, m}=\left[\left(1+\frac{1}{12} \delta_{y}^{2}\right) I+\frac{r}{2} A \delta_{y}^{2}\right] \mathbf{U}_{0, m}^{n+1}, \\
\dot{\mathbf{U}}_{L+1, m}=\left[\left(1+\frac{1}{12} \delta_{y}^{2}\right) I+\frac{r}{2} A \delta_{y}^{2}\right] \mathbf{U}_{L+1, m}^{n+1} .
\end{gathered}
$$

We can easily write a generalized version of D'Yakonov ADI like method for solving the two-dimensional coupled nonlinear Schrödinger system (1) as

$$
\begin{aligned}
{\left[\left(1+\sigma \delta_{x}^{2}\right) I+\frac{r}{2} A \delta_{x}^{2}\right] \dot{\mathbf{U}} } \\
=\left[\left(1+\sigma \delta_{x}^{2}\right) I-\frac{r}{2} A \delta_{x}^{2}\right]\left[\left(1+\sigma \delta_{y}^{2}\right) I-\frac{r}{2} A \delta_{y}^{2}\right] \mathbf{U}_{l, m}^{n} \\
-k\left(1+\sigma \delta_{x}^{2}\right)\left(1+\sigma \delta_{y}^{2}\right) B(\overline{\mathbf{U}}) \overline{\mathbf{U}} \\
\\
\quad\left[\left(1+\sigma \delta_{y}^{2}\right) I+\frac{r}{2} A \delta_{y}^{2}\right] \mathbf{U}_{l, m}^{n+1}=\dot{\mathbf{U}}
\end{aligned}
$$

for arbitrary value of $\sigma$, and for $\sigma=0, \sigma=1 / 12$ we recover the second- and fourth-order schemes, respectively.

\section{Stability Analysis}

To study the stability of the proposed scheme, we consider the von Neumann stability analysis which can be only applied for the linear finite difference scheme, so we consider the linear version of the generalized scheme

$$
\begin{aligned}
{\left[\left(1+\sigma \delta_{x}^{2}\right) I+\frac{r}{2} A \delta_{x}^{2}\right]\left[\left(1+\sigma \delta_{y}^{2}\right) I+\frac{r}{2} A \delta_{y}^{2}\right] \mathbf{U}_{l, m}^{n+1} } \\
=\left[\left(1+\sigma \delta_{x}^{2}\right) I-\frac{r}{2} A \delta_{x}^{2}\right]\left[\left(1+\sigma \delta_{y}^{2}\right) I-\frac{r}{2} A \delta_{y}^{2}\right] \mathbf{U}_{l, m}^{n} \\
\quad-\frac{k}{2} \omega\left(1+\sigma \delta_{x}^{2}\right)\left(1+\sigma \delta_{y}^{2}\right) \overline{\mathbf{U}}
\end{aligned}
$$

where $\omega$ is constant and $\sigma=0,1 / 12$. 


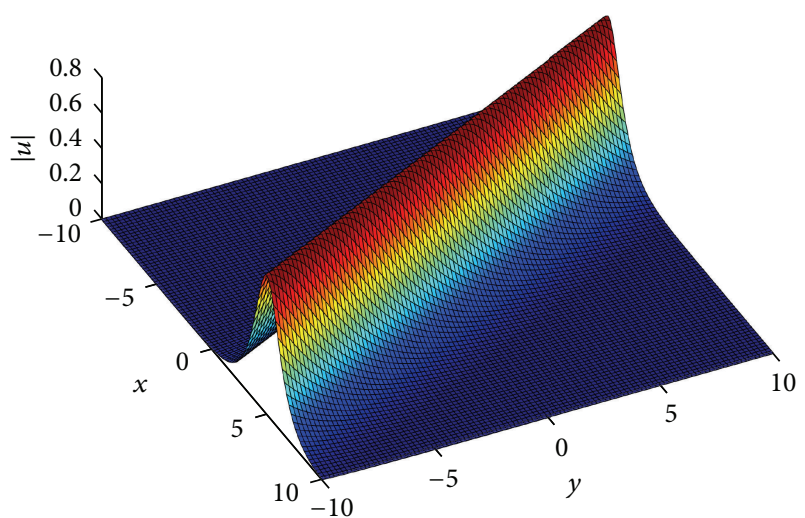

FIGURE 1: Single soliton $\beta_{1}=\kappa_{1}=0.5, \beta_{2}=\kappa_{2}=1, \alpha=1, t=0$.

Suppose that the numerical solution can be expressed by Fourier series, whose typical term is

$$
\mathbf{U}_{l, m}^{n}=G^{n} \mathbf{W}^{n} \exp \left(i \beta_{1} l h+i \beta_{2} m h\right)
$$

where $i=\sqrt{-1}, G^{n}$ is the amplification matrix at time level $n$, and $\beta_{1}, \beta_{2}$ are the wave numbers in $x$ and $y$ directions. Substituting (40) into (39) will lead to the matrix equation

$$
\begin{aligned}
& {\left[I-2 r \mu_{x} A\right]\left[I-2 r \mu_{y} A\right] G} \\
& \quad=\left[I+2 r \mu_{x} A\right]\left[I+2 r \mu_{y} A\right]-\frac{k}{2} \omega[G+I],
\end{aligned}
$$

where

$$
\mu_{x}=\sin ^{2}\left(\beta_{1} l h\right), \quad \mu_{y}=\sin ^{2}\left(\beta_{2} m h\right) .
$$

The eigenvalues of the matrix $G$ are given by

$$
\text { eigenvalues of } G=\frac{\left[1+2 i r \mu_{x}\right]\left[1+2 i r \mu_{y}\right]-(k / 2) \omega}{\left[1-2 i r \mu_{x}\right]\left[1-2 i r \mu_{y}\right]+(k / 2) \omega}
$$

It can be easily shown that the modulus of the maximum eigenvalue of the amplification matrix $G$ is less than one; hence, the scheme is unconditionally stable according to the von Neumann stability analysis.

\section{Numerical Results}

In this section, the efficiency and accuracy of the proposed schemes will be tested by comparing with the exact solutions. We will measure the accuracy of the proposed schemes using the $L_{\infty}$ norm. We compute the conserved quantity by using the trapezoidal rule.

4.1. Example 1. In this example, we choose the initial conditions from the exact solutions

$$
\begin{aligned}
& \psi(x, y, t)=A \operatorname{sech}(\xi) \exp (i \eta), \\
& \phi(x, y, t)=B \operatorname{sech}(\xi) \exp (i \eta),
\end{aligned}
$$

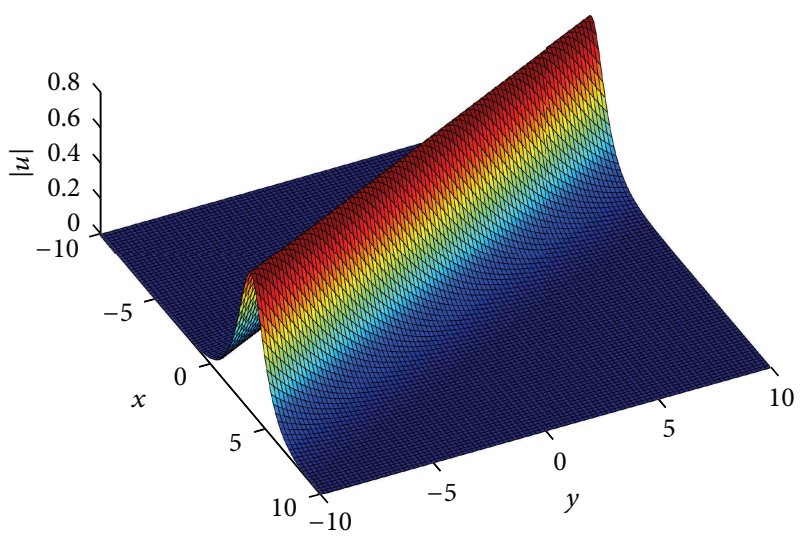

FIGURE 2: Single soliton $\beta_{1}=\kappa_{1}=0.5, \beta_{2}=\kappa_{2}=1, \alpha=1, t=1$.

TABLE 1: Single soliton with $\sigma=0$.

\begin{tabular}{lccc}
\hline Iter & $T$ & ER & $I$ \\
\hline 0 & 0 & 0 & 24.99989 \\
2 & 0.2 & 0.00435 & 25.00086 \\
2 & 0.4 & 0.00886 & 25.00291 \\
2 & 0.6 & 0.01365 & 25.00586 \\
2 & 0.8 & 0.01893 & 25.00973 \\
2 & 1.0 & 0.02439 & 25.01458 \\
\hline
\end{tabular}

where

$$
\begin{gathered}
\xi=\beta_{1} x+\beta_{2} y-v t, \quad \eta=-\kappa_{1} x-\kappa_{2} y+\omega t+\theta, \\
A=B=\left[\frac{2 \delta\left(\beta_{1}^{2}+\beta_{2}^{2}\right)}{(1+\alpha)}\right]^{1 / 2},
\end{gathered}
$$

at $t=0$. The following parameters are used

$$
\begin{gathered}
h=0.2, \quad k=0.001, \quad \beta_{1}=0.5, \\
\beta_{2}=1.0, \quad k_{1}=0.5, \quad k_{2}=1.0, \\
\alpha=1, \quad \delta=\frac{1}{2}, \quad-10 \leq x, y \leq 10 .
\end{gathered}
$$

The boundary conditions are extracted from the exact solution (44).

Tables 1, 2, and 3 show the errors (ER) and the conserved quantities (I) for $\sigma=0,1 / 6$ and $\sigma=1 / 12$, respectively. We have noticed that the scheme with $\sigma=1 / 12$ produced highly accurate results, and this is due to the fourth order accuracy in space and second-order accuracy in time. The other two methods using $\sigma=0$ and $\sigma=1 / 6$ are of second-order accuracy in space and time. Figure 1 shows a single soliton at $t=0$. Figure 2 shows the numerical solution at $t=1$. 


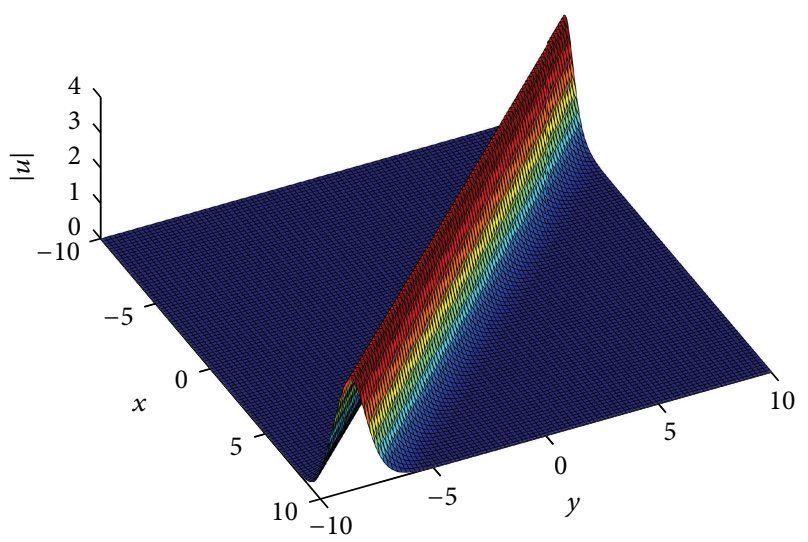

FIGURE 3: Soliton solution $|\psi|^{2}$ at $t=0$.

TABLE 2: Single soliton with $\sigma=1 / 6$.

\begin{tabular}{lccc}
\hline Iter & $T$ & ER & $I$ \\
\hline 0 & 0 & 0 & 24.99989 \\
2 & 0.2 & 0.00436 & 24.99882 \\
2 & 0.4 & 0.00859 & 24.99664 \\
2 & 0.6 & 0.01277 & 24.99351 \\
2 & 0.8 & 0.01750 & 24.98938 \\
2 & 1.0 & 0.02206 & 24.98417 \\
\hline
\end{tabular}

TABLE 3: Single soliton with $\sigma=1 / 12$.

\begin{tabular}{lccc}
\hline Iter & $T$ & $\mathrm{ER}$ & $I$ \\
\hline 0 & 0 & 0 & 24.99989 \\
2 & 0.2 & 0.00011 & 24.99989 \\
2 & 0.4 & 0.00018 & 24.99987 \\
2 & 0.6 & 0.00025 & 24.99981 \\
2 & 0.8 & 0.00030 & 24.99971 \\
2 & 1.0 & 0.00036 & 24.99952 \\
\hline
\end{tabular}

4.2. Example 2. In this example we will choose the initial condition [1]

$$
\begin{aligned}
\psi(x, y, 0)= & \frac{\alpha_{1}}{2} \exp \left(\frac{-\eta}{2}\right) \text { sech } \\
& \times\left[\operatorname{Re}\left(\kappa_{1} x+l_{1} y\right)+\frac{\eta}{2}\right] \exp \left(i \operatorname{Im}\left(\kappa_{1} x+l_{1} y\right)\right) \\
\phi(x, y, 0)= & \frac{\beta_{1}}{2} \exp \left(\frac{-\eta}{2}\right) \operatorname{sech} \\
& \times\left[\operatorname{Re}\left(\kappa_{1} x+l_{1} y\right)+\frac{\eta}{2}\right] \exp \left(i \operatorname{Im}\left(\kappa_{1} x+l_{2} y\right)\right),
\end{aligned}
$$

where

$$
\exp (\eta)=\frac{1}{2} \frac{\left|\alpha_{1}\right|^{2}+\left|\beta_{1}\right|^{2}}{\left(\kappa_{1}+\kappa_{1}^{*}\right)^{2}+\left(l_{1}+l_{1}^{*}\right)^{2}}
$$

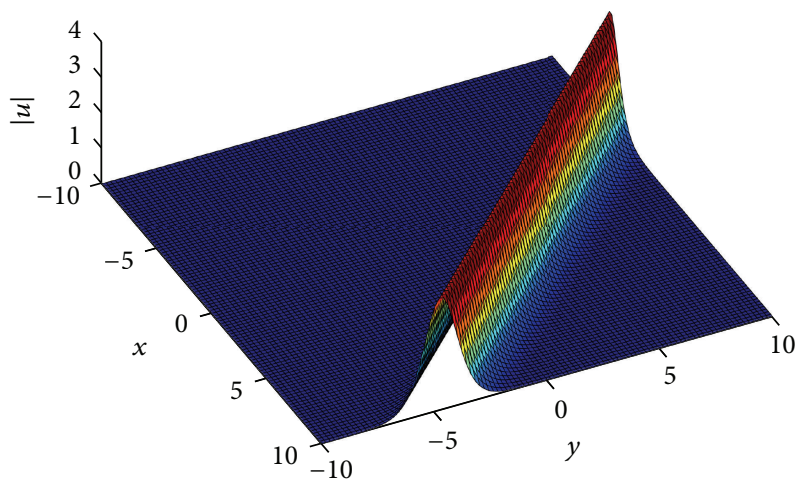

FIGURE 4: Soliton solution $|\psi|^{2}$ at $t=1$.

which represent two solitons of different amplitudes. The parameters $\alpha_{1}, \beta_{1}, \kappa_{1}$, and $l_{1}$ are complex parameters. In this test, we choose the parameters

$$
\begin{gathered}
h=0.2, \quad k=0.01, \quad x_{l}=-10, \quad \alpha_{1}=1, \\
\beta_{1}=0.5, \quad \kappa_{1}=l_{1}=1+i .
\end{gathered}
$$

In Figures 3 and 4, we display the numerical solution of $|\psi|^{2}$ at $t=0$ and $t=1$, while in Figures 5 and 6 we display the numerical solution of $|\phi|^{2}$ at $t=0$ and $t=1$.

4.3. Example 3. To study the interaction of two solitons, many numerical tests have been conducted with different initial conditions, and, among these tests, we select the initial conditions of the form

$$
\begin{aligned}
& \psi(x, y, 0) \\
& \quad=A \exp \left(-(x-d)^{2}-y^{2}\right) \exp \left(-i \ln \left(\cosh \left(x^{2}+y^{2}\right)\right)\right), \\
& \phi(x, y, 0) \\
& \quad=B \exp \left(-(x+d)^{2}-y^{2}\right) \exp \left(-i \ln \left(\cosh \left(x^{2}+y^{2}\right)\right)\right),
\end{aligned}
$$

which represent two solitons moving in the opposite direction and centered at $d$ and $-d$, respectively. In this test we choose the parameters

$$
\begin{gathered}
h=0.2, \quad k=0.01, \quad x_{l}=-10, \quad d=5.0, \\
A=1, \quad B=0.8, \quad t=0, \ldots, 1.5 .
\end{gathered}
$$

The interaction scenario is given in Figures 7 and 8 . In Figure 7 we show the two solitons with two different amplitudes at $t=0$. In Figure 8 we display the interaction where the two solitons interact at $t=0.5$, and, in Figure 9, we display the two solitons after the interaction at $t=1.5$. In Table 4, we display the conserved quantities, and we see that the numerical method we proposed conserves the conserved quantities almost exactly. It is easy to see that the interaction regiem is an inelastic one. See [18]. 


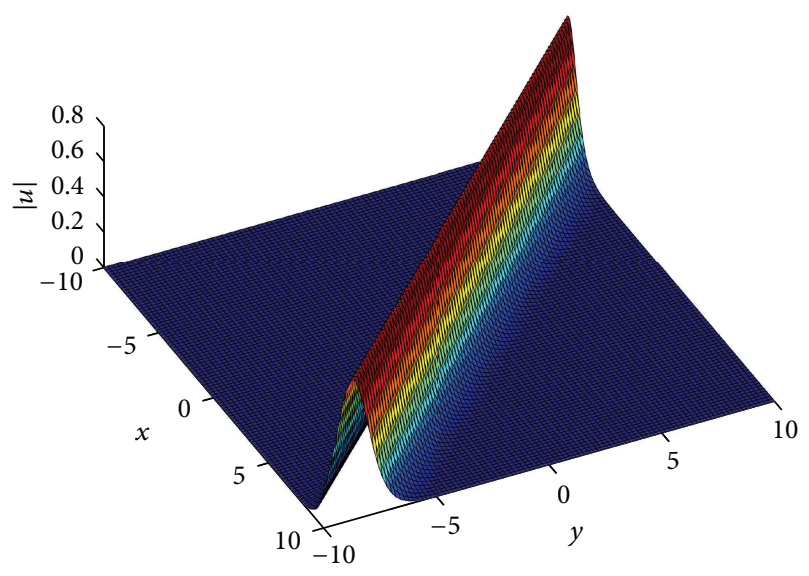

FIgURE 5: Soliton solution $|\phi|^{2}$ at $t=0$.

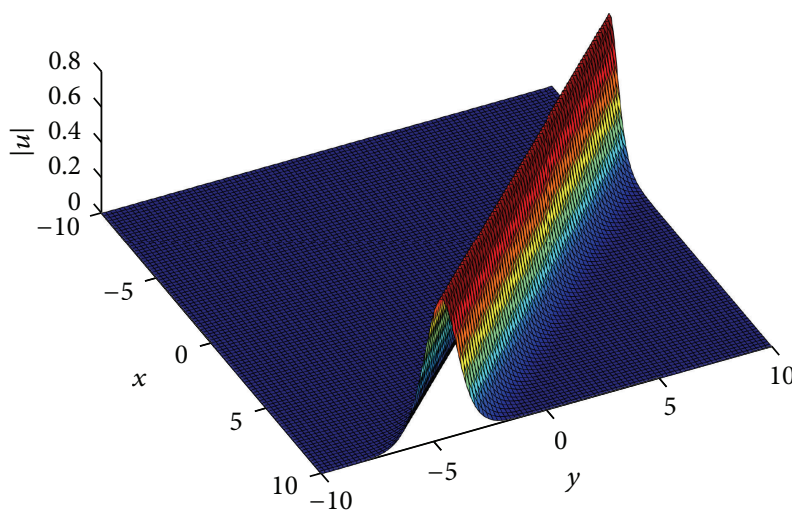

FIgURE 6: Soliton solution $|\phi|^{2}$ at $t=1$.

4.4. Example 4. The system under consideration generates a progressive plane wave solutions [7]:

$$
\begin{aligned}
& \psi(x, y, t)=A \exp \left(i\left(\kappa_{1} x+\kappa_{2} y+\omega t\right)\right), \\
& \phi(x, y, t)=A \exp \left(i\left(\kappa_{1} x+\kappa_{2} y+\omega t\right)\right),
\end{aligned}
$$

where

$$
\omega=k_{1}^{2}+k_{2}^{2}-(1+\alpha)|A|^{2} .
$$

Our numerical experiments are conducted in the domain $[0,2 \pi] \times[0,2 \pi]$ with

$$
\begin{gathered}
A=1, \quad \alpha=1, \quad k_{1}=k_{2}=1, \quad \delta=1, \\
k=0.001, \quad h=\frac{\pi}{50} .
\end{gathered}
$$

Initial and boundary conditions are extracted from the exact solution. In this example we choose $\sigma=1 / 12$, the fourth order ADI method. The numerical results are presented in Table 5. Figure 10 displays the solution at $t=0$, and Figure 11 displays, the solution at $t=1$.

Table 4 displays the accuracy of the scheme and preserves the conserved quantities.

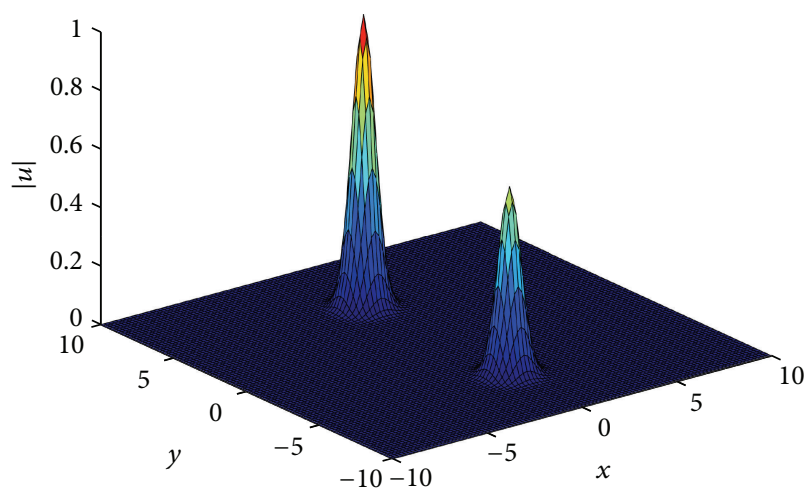

Figure 7: Interaction of two solitons $|\psi|^{2}$ and $|\phi|^{2}$ at $t=0$.

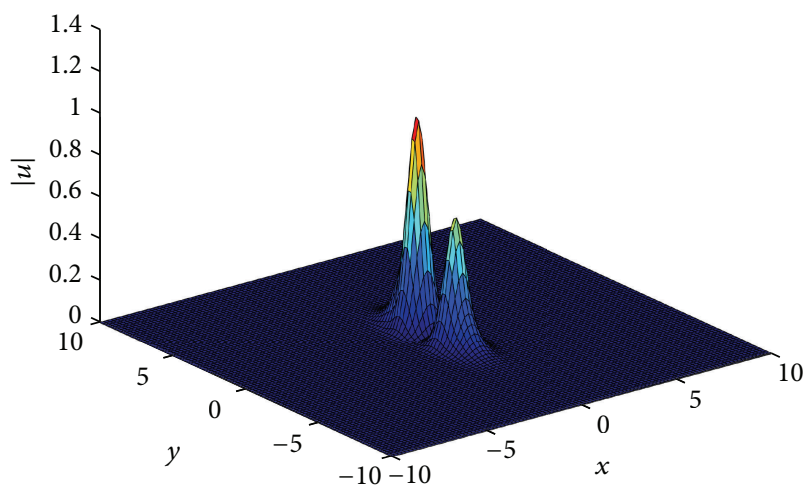

Figure 8: Interaction of two solitons $|\psi|^{2}$ and $|\phi|^{2}$ at $t=0.5$.

TABLE 4: The conserved quantities during the interaction scenario.

\begin{tabular}{lcc}
\hline$T$ & $I_{1}$ & $I_{2}$ \\
\hline 0.0 & 1.57080 & 1.00531 \\
0.5 & 1.57086 & 1.00533 \\
1.0 & 1.57065 & 1.00522 \\
1.5 & 1.57060 & 1.00520 \\
\hline
\end{tabular}

TABle 5: Periodic solution $k_{1}=k_{2}=\alpha=1$.

\begin{tabular}{lccc}
\hline Iter & $T$ & ER & $I$ \\
\hline 0 & 0.0 & 0.000000 & 39.478420 \\
2 & 0.2 & 0.000003 & 39.478420 \\
2 & 0.4 & 0.000005 & 39.478420 \\
2 & 0.6 & 0.000007 & 39.478420 \\
2 & 0.8 & 0.000009 & 39.478420 \\
2 & 1.0 & 0.000008 & 39.478420 \\
\hline
\end{tabular}

\section{Parallel Algorithm for the Proposed ADI Schemes}

It is to be noted that the implementation of the ADI schemes requires solving the same block-tridiagonal matrix with different right-hand sides. This can be done efficiently by using the fast parallel algorithm given in [19]. This algorithm 


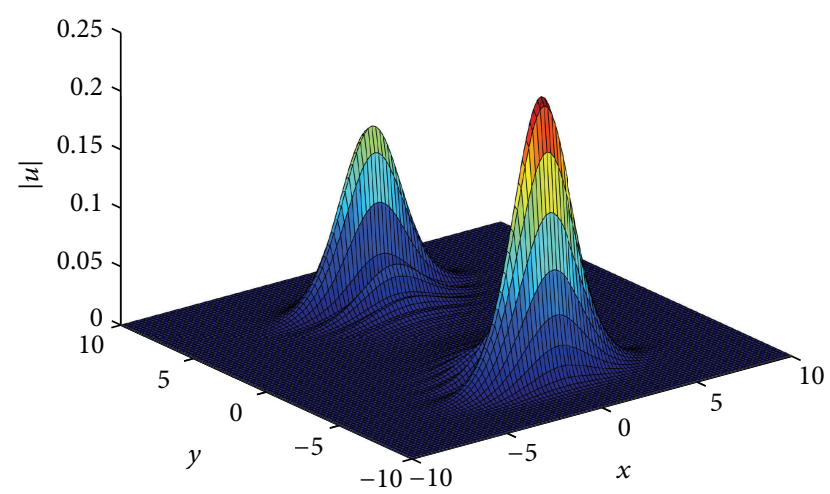

FIGURE 9: Interaction of two solitons $|\psi|^{2}$ and $|\phi|^{2}$ at $t=1.5$.

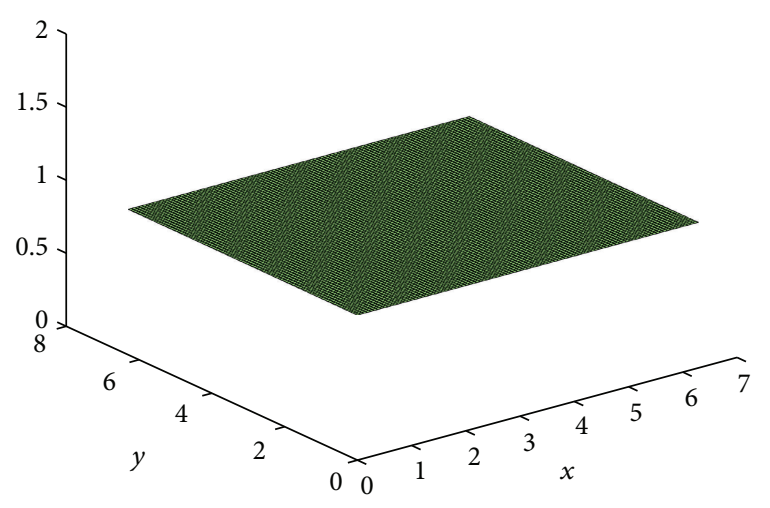

FIGURE 10: Initial condition with $k_{1}=k_{2}=\alpha=1$, at $t=0$.

[19] is a generalization of the parallel dichotomy algorithm for solving tridiagonal liner system of equations [20].

It has been shown that this dichotomy yields almost a linear speedup on a high performance system with many processors [19]. We expect the same speedup for our proposed methods. The parallel implementation of the proposed ADI method will be reported in our future work.

\section{Concluding Remarks}

In this present work, a generalized alternating direction implicit methods for solving two-dimensional coupled nonlinear Schrödinger equation have been established, the methods are unconditionally stable. The methods produced schemes of second and fourth order accuracy in space and second order in time according to the selected value of $\sigma$. The numerical results have shown that the proposed schemes successfully combine accuracy and efficiency for the two-dimensional coupled nonlinear Schrödinger system. The implementation of the ADI schemes requires solving the same block-tridiagonal matrix with different right-hand sides. This can be done by using the fast parallel algorithm given in [19]. This algorithm yields almost a linear speedup on a high performance system with many processors [19]. We expect the same speedup for our proposed methods. The proposed schemes can be easily extended to solve higher dimensional coupled nonlinear Schrödinger system.

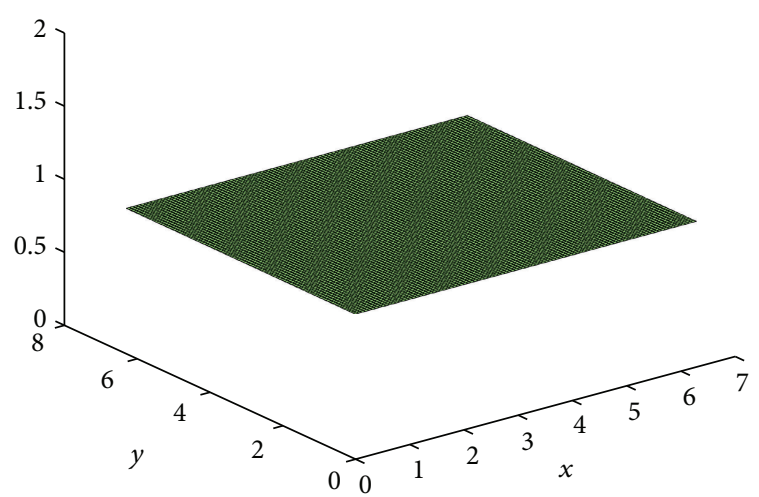

FIGURE 11: Numerical solution with $k_{1}=k_{2}=\alpha=1$, at $t=1$.

\section{Appendix}

The exact solution of the nonlinear system can be derived as follows: we assume [19]

$$
\begin{aligned}
& \psi(x, y, t)=A \operatorname{sech}(\xi) \exp (i \eta), \\
& \phi(x, y, t)=B \operatorname{sech}(\xi) \exp (i \eta),
\end{aligned}
$$

where

$$
\begin{gathered}
\xi=\beta_{1} x+\beta_{2} y-v t, \\
\eta=-\kappa_{1} x-\kappa_{2} y+\omega t+\theta .
\end{gathered}
$$

Now from (A.1), we deduce

$$
\begin{aligned}
& \psi_{t}=[v A \tanh (\xi) \operatorname{sech}(\xi)+i \omega A \operatorname{sech}(\xi)] \exp (i \eta), \\
& \psi_{x x}=\left[A \beta_{1}^{2} \operatorname{sech}(\xi)-2 A \beta_{1}^{2} \operatorname{sech}^{3}(\xi)-A \kappa_{1}^{2} \operatorname{sech}(\xi)\right. \\
& \left.+2 i \kappa_{1} \beta_{1} A \tanh (\xi) \operatorname{sech}(\xi)\right] \exp (i \eta), \\
& \psi_{y y}=\left[A \beta_{2}^{2} \operatorname{sech}(\xi)-2 A \beta_{2}^{2} \operatorname{sech}^{3}(\xi)-A \kappa_{2}^{2} \operatorname{sech}(\xi)\right. \\
& \left.+2 i \kappa_{2} \beta_{2} A \tanh (\xi) \operatorname{sech}(\xi)\right] \exp (i \eta),
\end{aligned}
$$

and similar expressions can be obtained for the function $\phi$. By substituting these expressions into the given system, and equating the real and imaginary parts, this will produce the following relations:

$$
\begin{gathered}
v=-2 \delta\left(\kappa_{1} \beta_{1}+\kappa_{2} \beta_{2}\right), \\
-\omega A \operatorname{sech}(\xi)+\delta A \beta_{1}^{2} \operatorname{sech}(\xi)-2 \delta A \beta_{1}^{2} \operatorname{sech}^{3}(\xi) \\
-\delta A \kappa_{1}^{2} \operatorname{sech}(\xi)+\delta A \beta_{2}^{2} \operatorname{sech}(\xi) \\
-2 \delta A \beta_{2}^{2} \operatorname{sech}^{3}(\xi)-\delta A \kappa_{2}^{2} \operatorname{sech}(\xi) \\
+\left(A^{2}+\alpha B^{2}\right) A \operatorname{sech}^{3}(\xi)=0 .
\end{gathered}
$$

By equating the coefficient of $\operatorname{sech}^{3}(\xi)$ in (A.5), we obtain

$$
A^{2}+\alpha B^{2}=2 \delta\left(\beta_{1}^{2}+\beta_{2}^{2}\right)
$$


and by equating the coefficients of $\operatorname{sech}(\xi)$, we will get the relation

$$
\omega=\delta\left[\left(\beta_{1}^{2}+\beta_{2}^{2}\right)-\left(\kappa_{1}^{2}+\kappa_{2}^{2}\right)\right] .
$$

From the second equation of the proposed system $(\phi)$, we will get the relation

$$
\alpha A^{2}+B^{2}=2 \delta\left(\beta_{1}^{2}+\beta_{2}^{2}\right)
$$

and from (A.6) and (A.8), we deduce that $A=B$, and then we get the relation

$$
A=B=\left[\frac{2 \delta\left(\beta_{1}^{2}+\beta_{2}^{2}\right)}{(1+\alpha)}\right]^{1 / 2}
$$

with $\alpha, \beta, \kappa$, and $l$ as arbitrary complex parameters.

\section{Acknowledgments}

This work was funded by the Deanship of Scientific Research (DSR), King Abdulaziz University, Jeddah, under Grant no. (130-074-D1433). The authors, therefore, acknowledge with thanks the DSR technical and financial support.

\section{References}

[1] H.-Q. Zhang, X.-H. Meng, T. Xu, L.-L. Li, and B. Tian, "Interactions of bright solitons for the $(2+1)$-dimensional coupled nonlinear Schrödinger equations from optical fibres with symbolic computation," Physica Scripta, vol. 75, no. 4, pp. 537-542, 2007.

[2] M. S. Ismail and T. R. Taha, "Numerical simulation of coupled nonlinear Schrödinger equation," Mathematics and Computers in Simulation B, vol. 56, no. 6, pp. 547-562, 2001.

[3] M. S. Ismail, "A fourth-order explicit schemes for the coupled nonlinear Schrödinger equation," Applied Mathematics and Computation, vol. 196, no. 1, pp. 273-284, 2008.

[4] M. S. Ismail, "Numerical solution of coupled nonlinear Schrödinger equation by Galerkin method," Mathematics and Computers in Simulation, vol. 78, no. 4, pp. 532-547, 2008.

[5] M. S. Ismail and S. Z. Alamri, "Highly accurate finite difference method for coupled nonlinear Schrödinger equation," International Journal of Computer Mathematics, vol. 81, no. 3, pp. 333351, 2004.

[6] T. R. Taha and X. Xu, "Parallel split-step fourier methods for the coupled nonlinear Schrödinger type equations," The Journal of Supercomputing, vol. 32, no. 1, pp. 5-23, 2005.

[7] Z. Gao and S. Xie, "Fourth-order alternating direction implicit compact finite difference schemes for two-dimensional Schrödinger equations," Applied Numerical Mathematics, vol. 61, no. 4, pp. 593-614, 2011.

[8] L. Kong, Y. Duan, L. Wang, X. Yin, and Y. Ma, "Spectrallike resolution compact ADI finite difference method for the multi-dimensional Schrödinger equations," Mathematical and Computer Modelling, vol. 55, no. 5-6, pp. 1798-1812, 2012.

[9] Z. F. Tian and P. X. Yu, "High-order compact ADI (HOCADI) method for solving unsteady 2D Schrödinger equation," Computer Physics Communications, vol. 181, no. 5, pp. 861-868, 2010.
[10] T. Wang, B. Guo, and Q. Xu, "Fourth order compact and energy conservative difference schemes for the nonlinear Schrödinger equation in two dimensions," Journal of Computational Physics, vol. 243, pp. 382-399, 2013.

[11] Y. Xu and L. Zhang, "Alternating direction implicit method for solving two-dimensional cubic nonlinear Schrödinger equation," Computer Physics Communications, vol. 183, no. 5, pp. 1082-1093, 2012.

[12] S. Karaa and J. Zhang, "High order ADI method for solving unsteady convection-diffusion problems," Journal of Computational Physics, vol. 198, no. 1, pp. 1-9, 2004.

[13] M. Sapagovas and K. Jakubeliene, "Alternating direction method for two-dimensional parabolic equation with nonlocal integral condition," Nonlinear Analysis, vol. 17, no. 1, pp. 91-98, 2012.

[14] M. Subasi, "On the finite difference schemes for the numerical solution of two dimensional Schrödinger equation," Numerical Methods for Partial Differential Equations, vol. 18, pp. 124-134, 2002.

[15] D. You, "A high-order Padé ADI method for unsteady convection-diffusion equations," Journal of Computational Physics, vol. 214, no. 1, pp. 1-11, 2006.

[16] A. Biswas, "1-soliton solution of $1+2$ dimensional nonlinear Schrödinger equations in power law media," Communications in Nonlinear Science and Numerical Simulation, vol. 14, pp. 1830$1833,2009$.

[17] A. R. Mitchell and D. F. Griffiths, The Finite Difference Method in Partial Differential Equations, John Wiley \& Sons, Chichester, UK, 1980.

[18] C. Besse, N. J. Mauser, and H. P. Stimming, "Numerical studies for nonlinear Schrodinger equations: the Schrodinger-Poisson$\mathrm{X} \alpha$ model and Davey Stewartson systems," In press.

[19] A. V. Terekhov, "A fast parallel algorithm for solving blocktridiagonal systems of liner equations including the domain decomposition method," Parallel Computing, vol. 39, pp. 245258, 2013.

[20] A. V. Terekhov, "Parallel dichotomy algorithm for solving tridiagonal system of linear equations with multiple right-hand sides," Parallel Computing, vol. 36, no. 8, pp. 423-438, 2010. 


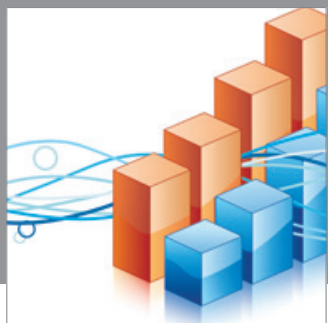

Advances in

Operations Research

mansans

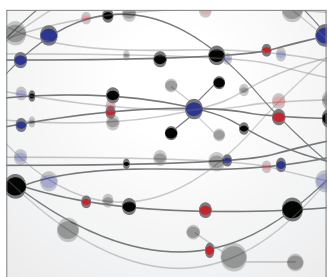

The Scientific World Journal
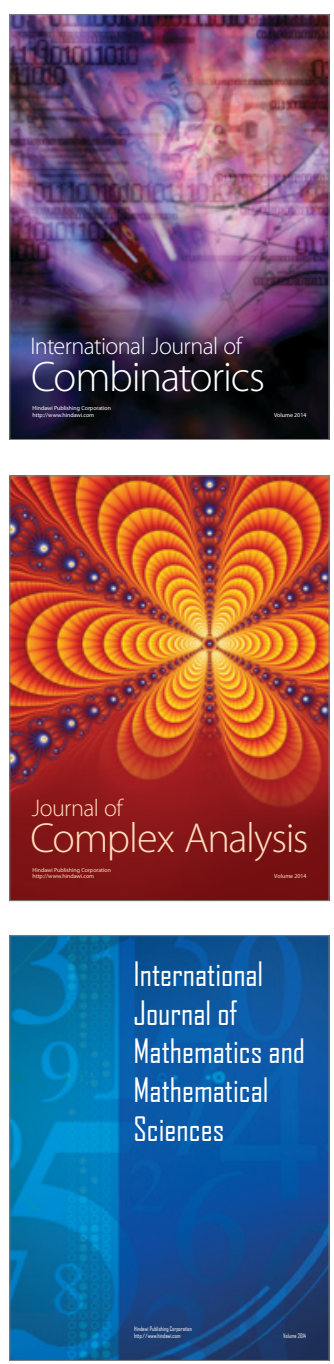
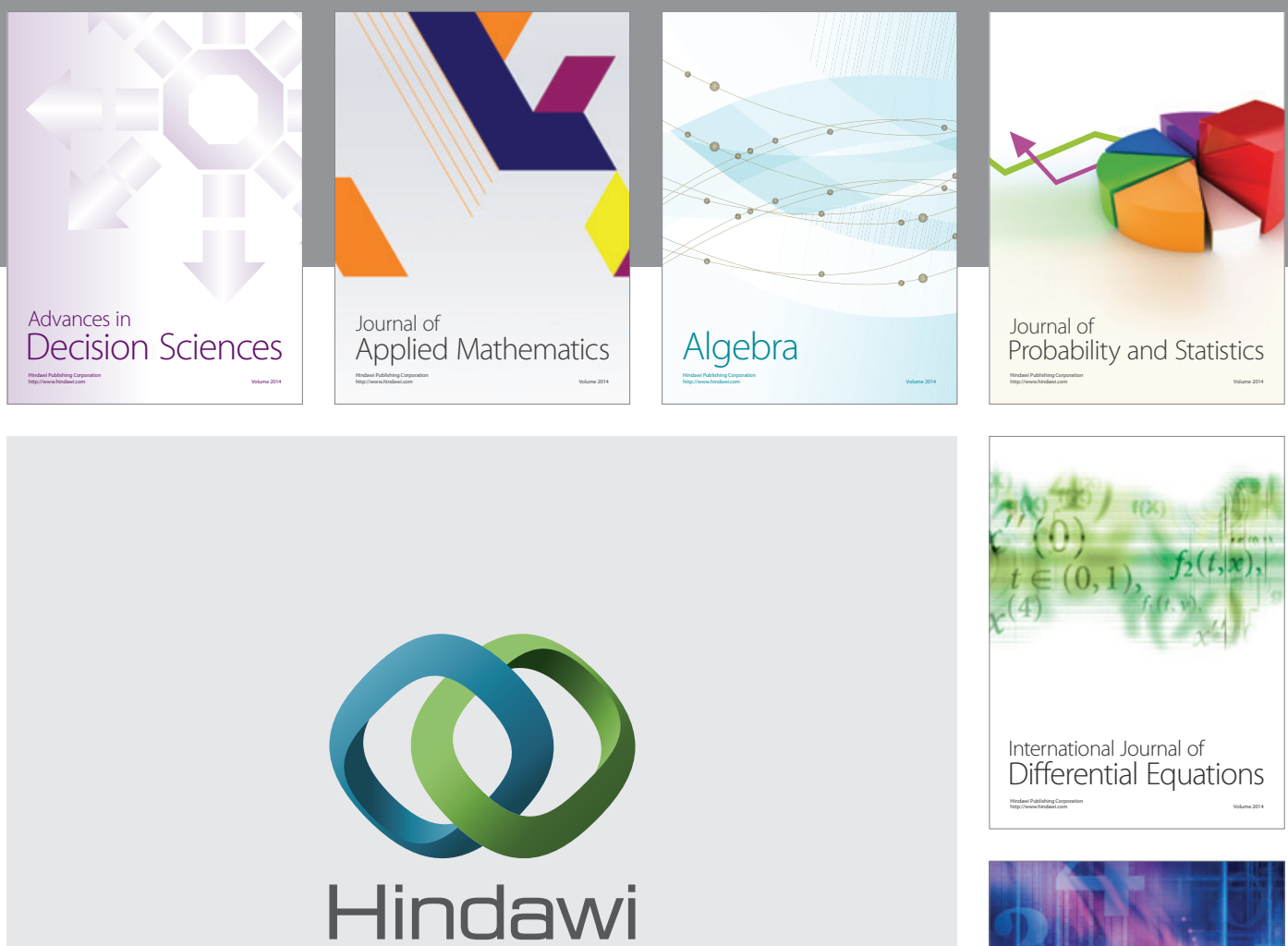

Submit your manuscripts at http://www.hindawi.com
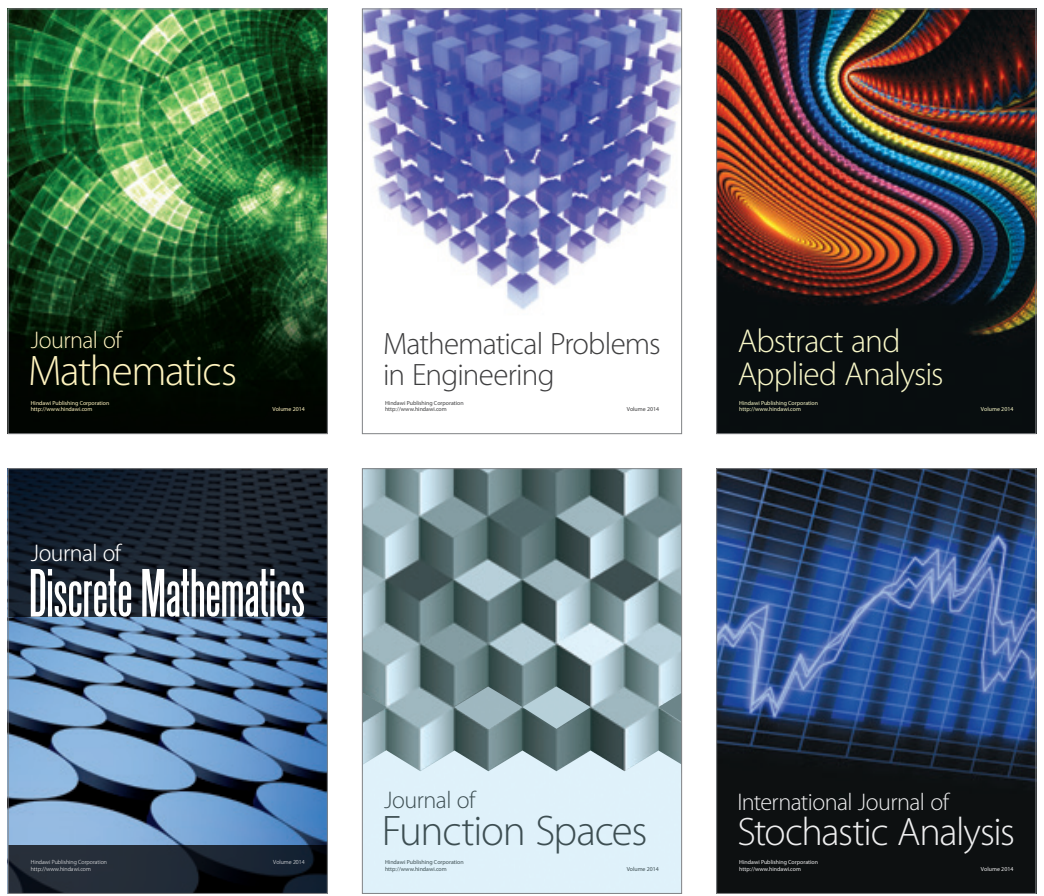

Journal of

Function Spaces

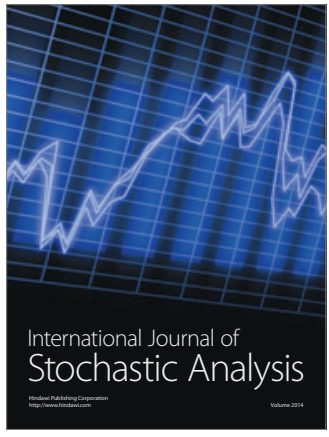

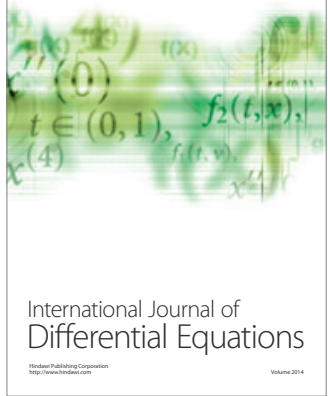
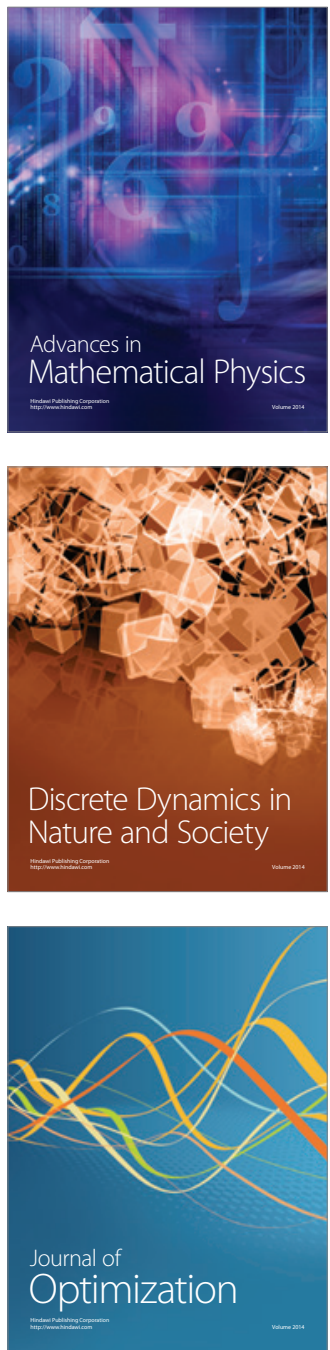